\title{
EXPERIMENTAL MEASUREMENT OF HIGH-GRADIENT STANDING WAVE ACCELERATOR TRANSPORT MATRIX
}

\author{
S. Reiche, DESY, Hamburg, Germany \\ J.B. Rosenzweig, University of California, Los Angeles, USA \\ L. Serafini, INFN-Milano, Milan, Italy
}

Abstract

This paper presents an experimental study of the transverse beam dynamics of an electron beam in a high-gradient, standing wave linear accelerator. A $3.6 \mathrm{MeV}$ beam from the UCLA RF Photoinjector (SATURNUS) is injected at various phases and $\mathrm{rf}$ field amplitudes into the plane wave transformer (PWT) linac (peak acceleration $40 \mathrm{MeV} / \mathrm{m}$ ), and its transverse dynamics measured by a corrector magnet sweeping method. This method allows us to reconstruct the transverse matrix elements, which are compared to analytical predictions(J.B. Rosenzweig and L.Serafini, Physical Review E 49, 1599 (1994)). The determinant of the experimentally derived matrix is found to be the ratio of the initial to final momentum, verifying the theory, and providing direct evidence of adiabatic damping of transverse trace space.

\section{INTRODUCTION}

With the rise in use of high gradient radio-frequency linear accelerators ( $\mathrm{rf}$ linacs), in devices such as $\mathrm{rf}$ photoinjectors[1] and linear collider test facilities[2], there has been increased attention placed on the strong transverse focusing effects present in these devices. These effects, which are due both to first order transient effects at the entrance and exit of a linac, and to second order ponderomotive (alternating gradient) effects in the body of the periodic linac structure, are of primary importance in understanding the beam transport in moderate energy sections $\left(5<\gamma=E / m_{e} c^{2}<100\right)$ of electron accelerators. While theoretical analyses of the focusing properties of linacs date back to the 1960 's[3, 4], recent work has produced a more detailed understanding of the ponderomotive force[5], and analytical solutions of these equations for arbitrary acceleration phase and spatial harmonic content of the rf fields have been found[6], which led to a matrix description of the trace space transport.

This matrix treatment of beam dynamics in high gradient rf linacs, as well as the underlying analytical model for the averaged (over an rf period) transverse forces, have formed the underpinning of much recent work, from the optics of linear collider test facilities[2], to the full theory of spacecharge dominated beam dynamics in rf photoinjectors[7]. While these implementations of the theory have been compared positively with computer simulation, there has, however, been no effort to date to verify the theoretical advances with experiment. This paper therefore presents a first such verification.

\section{THEORY}

The trace space transport matrix corresponding to a rf linac, which upon multiplication of a transverse trace space vector, e.g. $\left(x, x^{\prime}\right)$, gives the mapping of this vector through the linac, has recently been derived for arbitrary rf phase, amplitude, and spatial harmonic content in the linac. Including all terms to second order in the average accelerating gradient $e E_{0} \cos (\phi) \equiv \gamma^{\prime} m_{e} c^{2}$, where $E_{0}$ is the amplitude of the synchronous $\left(\nu_{\phi} \cong c\right)$ spatial harmonic wave component of the rf field, and $\phi=\omega t-k z$ is the phase defined with respect to the maximum acceleration in this wave, the action of a ponderomotive force can obtained to second order by averaging over the fast alternating gradient first order forces and the induced lowest order oscillatory motion, as $[5,6]$

$$
\overline{F_{r}}=\eta(\phi) \frac{\left(q E_{0}\right)^{2}}{8 \gamma m_{0} c^{2}} r
$$

with

$$
\eta(\phi) \equiv \sum_{n=1}^{\infty} b_{n}^{2}+b_{-n}^{2}+2 b_{n} b_{-n} \cos (2 \phi) .
$$

The coefficients $b_{n}$ are the Floquet amplitudes of the spatial harmonics, defined by the expression, valid for an ultrarelativistic $\left(\nu_{b} \cong c\right)$ electron

$$
E_{z} \equiv E_{0} \operatorname{Re}\left[\sum_{n=-\infty}^{\infty} b_{n} e^{i\left(2 k_{0} n z+\phi\right)}\right]
$$

where $k_{0}=\psi / d=\omega / c$ and $\psi$ is the rf phase shift per period of the linac, with $\psi=\pi$ in the structure considered here.

From the averaged force, the differential equation governing the seculat trajectory of the electron, about which the fast oscillations induced in first order are performed, is derived. Its general solutions, given in matrix form, can be found in Ref. 6. At the ends of an rf structure, there are first order transient angular kicks associated with entering and exiting the fringing field which are not immediately cancelled in first order by a nearby kick. These kicks, again valid when considering the secular trajectory, are given by $\pm \gamma^{\prime} / 2 \gamma$, for the exit and entrance regions, respectively. Multiplying the matrices associated with the entrance, interior, and exit of the linac, we have the full trace space transport matrix,

$$
\begin{gathered}
M= \\
{\left[\begin{array}{cc}
\cos (\alpha)-\sqrt{\frac{2}{\eta(\phi)}} \cos (\phi) \sin (\alpha) & \sqrt{\frac{8}{\eta(\phi)}} \frac{\gamma_{i}}{\gamma^{\prime}} \cos (\phi) \sin (\alpha) \\
-\frac{\gamma^{\prime}}{\gamma_{f}}\left[\frac{\sin (\phi)}{\sqrt{2 \eta(\phi)}}+\frac{\sqrt{\eta(\phi)}}{\sqrt{8} \cos (\phi)}\right] \sin (\alpha) & \frac{\gamma_{i}}{\gamma_{f}}\left[\cos (\alpha)+\sqrt{\frac{2}{\eta(\phi)}} \cos (\phi) \sin (\alpha)\right]
\end{array}\right]}
\end{gathered}
$$


Here $\alpha=\frac{\sqrt{\eta(\phi)}}{\sqrt{8} \cos (\phi)} \ln \left(\gamma_{f} / \gamma_{i}\right)$, with $\gamma_{i}$ and $\gamma_{f}$ the initial and final Lorentz factors of the electron, respectively, which are simply related by $\gamma_{f}=\gamma_{i}+\gamma^{\prime} L$, with $L$ equal to the length of the linac section, with $\gamma^{\prime} m_{e} c^{2}$ equal to the averaged (over a period of the linac) acceleration gradient.

\section{THE EXPERIMENT}

The linac used for the present experiments is a component of the UCLA Photoinjector, a rf accelerating section which boosts the energy of a $3.6 \mathrm{MeV}$ photoelectron beam extracted from a high gradient 1.5 cell $\pi$-mode rf gun to approximately $12.8 \mathrm{MeV}$ at maximum in these experiments. This linac is constructed of a novel, high shunt impedance design known as a plane-wave transformer[8] (PWT), and has non-negligible spatial harmonic content (e.g. $\eta(0)=$ 1.23). The experimental arrangement is nearly ideal for observing the focusing and adiabatic damping predicted by the matrix given in Eq. 4. This is due to a number of advantages, the first being that the beam can be viewed as essentially point-like for the purpose of centroid measurements, as it has a short rms phase extent, $\sigma_{\phi} \cong 1^{\circ}$ (as determined by coherent transition radiation[9] measurements), it has a low rms normalized emittance, measured as $\epsilon_{n}<$ $10^{-5} \mathrm{~m}$-rad, and moderately large charge per bunch, $Q \cong$ $200 \mathrm{pC}$. This charge is large enough to clearly observe the photoelectrons over the dark-current background, yet small enough that collective effects on the centroid motion, such as transverse wake-fields, can be neglected.

The additional advantage this arrangement offers is that the matrix elements to be measured are most strongly dependent on the ratio $\gamma_{f} / \gamma_{i}$, and on $\gamma^{\prime}$. The PWT is by nature a high gradient linac $\left(\gamma^{\prime} \leq 50 \mathrm{~m}^{-1}\right)$, with moderate length $L=42 \mathrm{~cm}$, and thus to obtain a large value $\gamma_{f} / \gamma_{i}$ one only need inject with a relatively small initial energy $\gamma_{i} m_{e} c^{2}$. This energy cannot, however, be made arbitrarily small due to the constraint that $\nu_{b} \cong c$, and a related requirement that the relative energy gain per period of the linac be much smaller than unity, both of which are required in order to guarantee the validity of Eq. 4. Thus the injection energy given by the rf gun (which must in fact produce a relativistic beam due to its extraction dynamics) is nearly ideal for maximizing the matrix elements given in Eq. 4 .

In order to determine the transfer matrix of the PWT linac, the centroid of the photo-electron beam is measured using a differential steering technique originally developed to calibrate the beam energy after the gun. For this measurement, the current $I$ of steering (or kicker) magnet $\mathrm{K} 1$ is swept while monitoring the centroid position of the electron beam on the downstream phosphor screen P1. The momentum of the beam is determined from the differential change of the centroid position $d x_{c} / d I$ with current $I$. The near-axis field $\int B_{y}(I, z) d z$ of the horizontally-deflecting steering magnets was determined with Hall probe measurements, and the phosphor screens and related video calibrations performed before the experiments.

This method of the momentum determination helps es- tablish the initial conditions of the beam, and also forms the technical basis for the matrix element measurements. As it is difficult to determine the actual electromagnetic center of the linac with respect to the insertable phosphor diagnostics, we were forced to use a differential steering technique. In this case, one of the low energy magnets previous to the linac (K1 or K2) was swept, while observing the centroid motion of the beam downstream of the linac (P2 or P3). The four combinations of the steering magnets and observation points, along with knowledge of the drift lengths, integrated field of the steering magnets, and precise calibration of the video images of the phosphor screens, give four measured quantities which allow reconstruction of the linac transport matrix.

\section{RESULTS}

In the initial round to experiments, the dependence of the matrix elements on linac injection phase was measured. For each phase, we swept through eight different currents in the steering magnets, and obtained a linear fit to the resulting centroid motion at the phosphor screens. Three noticeable effects were the source of small errors in this method: (1) a large amount of dark current emitted from the gun, (2) transverse centroid injection errors arising from cathode drive laser pointing jitter, and (3) a slow drift in the photocathode drive laser injection phase. This third effect allowed data to be taken over only a limited time span, with all data taken in a single session to ensure the reproducibility of conditions.

The data resulting from these measurements give the calculated values of the centroid sweep rate using the transport derived from the $M$-matrix and the relevant drift matrices. There is fairly good agreement for all four measurement sets between the predictions and data over all phases where reliable measurements could be made, with a slightly larger sensitivity on phase offsets from the crest displayed by the data. Note that there are larger errors associated with two measurements made at screen P3 for phases of -2.2 and +10 degrees. The P3 data was inherently more difficult to obtain, because the photoelectron beam was much larger in size than at P2, and the two more uncertain points suffered from large interference from the dark current background at their specific running conditions.

The data are inverted to give the elements of the $M$ matrix, with the results shown in Figs. 1. The relative experimental uncertainty shown by the error bars is larger than for the data sets due to the higher order sensitivity introduced by the inversion process. Also shown in Figs. 1 are the theoretical predictions for the $M$-matrix. Since the matrix elements were more sensitive to the experimental noise in the data, we also fit the data to a quadratic in $\phi$, and then used the resulting filtered data function in the inversion routine to generate a smooth fit in $\phi$ for the matrix elements. As can be seen, this fit agrees quite well with theoretical predictions.

As a final check on the validity of these data sets, and to independently verify the adiabatic damping component of 

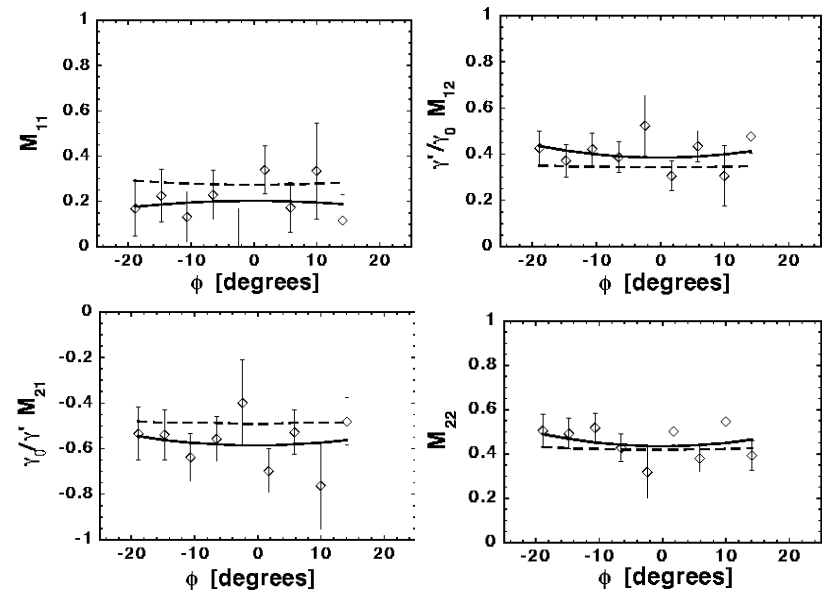

Figure 1: Matrix elements of the transverse trace space map $M$ of the PWT linac as a function of phase $\phi$. The values obtained from inverting a quadratic fit to the raw data are drawn by a solid line, the theoretical values by a dashed line. The matrix elements $M_{12}$ and $M_{21}$ are weighted by the factors $\gamma^{\prime} / \gamma_{0}$ and $\gamma_{0} / \gamma^{\prime}$, respectively, to normalize all matrix elements.

the transformation, in Fig. 2 (left) we display the experimentally derived values of $\operatorname{det}(M)$, as well as the predicted values, $\gamma_{i} / \gamma_{f}$. Because of the form of the algebraic relation between the determinant and the matrix elements, the relative experimental uncertainty in $\operatorname{det}(M)$ is no larger than for the elements themselves. We have also plotted the values of $\operatorname{det}(M)$ obtained from the quadratic fit to the raw data. Not too surprisingly, this produces agreement with theory as consistent as the agreement found for the matrix elements.
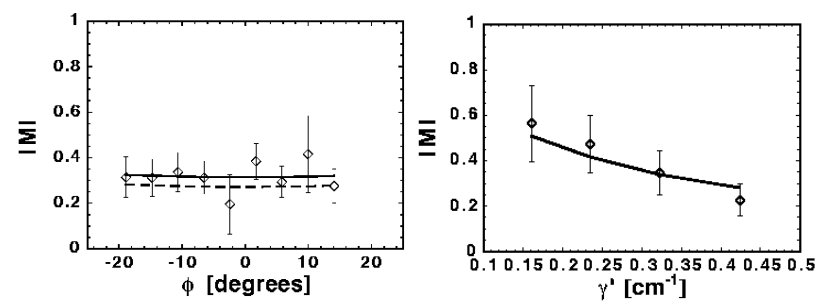

Figure 2: Determinant of the transverse trace space map for different acceleration phases $\phi$ (left) and gradients $\gamma^{\prime}$ (right). The values for the inverted fit to the data for the phase dependency are represented by a solid line, and for the theoretical predictions by a dashed line.

In order to complete a parametric study of the predictions of Eq. 4, and explore the adiabatic damping effects in more detail, we undertook a set of measurements of the matrix elements' dependence on $E_{0}$. In this case the rf attenuator was used to lessen the power fed into the linac, yielding, after resetting the phase to optimal acceleration, final beam energies of 7.1, 8.7, 10.6 and $12.8 \mathrm{MeV}$, or average accelerating fields of $E_{0}=8.3,12.1,16.6$, and $21.9 \mathrm{MV} / \mathrm{m}$, respectively. This data can then be inverted as before to give the matrix elements. These experimentally derived determinants are plotted in Fig. 2 (right), along with the predicted values of $\gamma_{i} / \gamma_{f}$. This plot, which shows the expected behavior quite well is, taken along with Fig. 2 (left), convincing direct evidence for the adiabatic damping of transverse "action", or area, in trace space.

\section{CONCLUSION}

In conclusion, these sets of measurements have provided a first verification of the theoretical model developed in Refs. 5 and 6 of linear transverse dynamics of electrons undergoing simultaneous strong acceleration as well as first and second order transverse focusing in an rf linac. The matrix treatment of the dynamics predicted by this model has been directly tested, with the experimentally derived matrix elements in fairly close agreement with the model derived elements. The determinants of the matrices obtained in this manner have been shown to display the expected dependence, $\operatorname{det}(M)=\gamma_{i} / \gamma_{f}$, giving direct evidence for the adiabatic damping of trace space area. Some deviations from theoretically predicted matrix elements themselves were noticeable, however, especially in the data obtained at lower acceleration gradient.

\section{ACKNOWLEDGMENTS}

This work performed with partial support from U.S. Dept. of Energy grants DE-FG03-90ER40796 and DE-FG0392ER40693, the Sloan Foundation grant BR-3225 and the Deutscher Akademischer Austauschdienst (German academic exchange program).

\section{REFERENCES}

[1] K.J. Kim, Nucl. Instruments and Methods A 275, 201 (1989).

[2] TESLA Test Facility Linac Conceptual Design Report, Ed.D. Edwards (DESY, Hamburg, 1995).

[3] E. Chambers, Stanford High Energy Physics Laboratory Report (Stanford, 1965).

[4] R. Helm and R. Miller, in Linear Accelerators, Eds. Pierre M. Lapostolle and Albert L. Septier (North-Holland, Amsterdam, 1969).

[5] S.C. Hartman and J.B. Rosenzweig, Phys. Rev. E 47, 2031 (1993).

[6] J.B. Rosenzweig and L. Serafini, Phys. Rev. E 49,1599 (1994).

[7] L. Serafini and J.B. Rosenzweig, TESLA report 96-12 (DESY, Hamburg, 1996), also submitted to Phys. Rev. E.

[8] R. Zhang, et. al., Proc. of the 1995 Part. Accel. Conference, 1102 (IEEE, 1995); D. Swenson, in Proc. of the 1988 European Part. Accel. Conf. p.1418 (World Scientific, 1989).

[9] U. Happek, A.J. Sievers, and E.B. Blum, Phys. Rev. Lett. 67, 2962 (1991). 